\title{
Tacrolimus (FK506) reduces ischemia-induced hippocampal damage in rats: a 7- and 30-day study
}

F. Giordani, A. Benetolli, L.A. Favero-Filho,

K.C.M. Lima, L. Cestari Junior and H. Milani
Departamento de Farmácia e Farmacologia, Centro de Ciências da Saúde, Universidade Estadual de Maringá, Maringá, PR, Brasil
Correspondence

H. Milani

Departamento de Farmácia e

Farmacologia, CCS, UEM

Av. Colombo, 5790

87020-900 Maringá, PR

Brasil

Fax: +55-44-263-6231

E-mail: hmilani@uem.br

Research supported by CNPq and UEM.

Received January 3, 2002 Accepted November 5, 2002

\begin{abstract}
The neuroprotective effect of the immunosuppressant agent FK506 was evaluated in rats after brain ischemia induced for $15 \mathrm{~min}$ in the 4vessel occlusion model. In the first experimental series, single doses of $1.0,3.0$ or $6.0 \mathrm{mg}$ FK506/kg were given intravenously (iv) immediately after ischemia. In the second series, FK506 $(1.0 \mathrm{mg} / \mathrm{kg})$ was given $i v$ at the beginning of reperfusion, followed by doses applied intraperitoneally (ip) $6,24,48$, and $72 \mathrm{~h}$ post-ischemia. The same protocol was used in the third series except that all 5 doses were given $i v$. Damage to the hippocampal field CA1 was assessed 7 or 30 days post-ischemia on three different stereotaxic planes along the septotemporal axis of the hippocampus. Ischemia caused marked neurodegeneration on all planes $(\mathrm{P}<0.001)$. FK506 failed to provide neuroprotection to CA 1 both when applied $i v$ as a single dose of 1.0, 3.0 or $6.0 \mathrm{mg} / \mathrm{kg}$ (experiment 1), and after five $i v$ injections of $1.0 \mathrm{mg} /$ $\mathrm{kg}$ (experiment 3 ). In contrast, the repeated administration of FK506 combining iv plus ip administration reduced CA1 cell death on all stereotaxic planes both 7 and 30 days post-ischemia (experiment 2; $\mathrm{P} \leq 0.01)$. Compared to vehicle alone, FK506 reduced rectal temperature in a dose-dependent manner $(\mathrm{P} \leq 0.05)$; however, this effect did not alter normothermia $\left(37^{\circ} \mathrm{C}\right)$. FK506 reduced ischemic brain damage, an effect sustained over time and apparently dependent on repeated doses and on delivery route. The present data extend previous findings on the rat 4-vessel occlusion model, further supporting the possible use of FK506 in the treatment of ischemic brain damage.
\end{abstract}

\section{Introduction}

Humans often suffer transient, global cerebral ischemia resulting from cardiocirculatory arrest or cardiopulmonary bypass surgery. In humans $(1,2)$ and in experimental animals $(3,4)$, brief interruptions of cerebral blood flow cause irreversible neuronal damage to vulnerable brain regions such as the
Key words

- Cerebral ischemia

- Hippocampal cell death

- FK506

- Sustained neuroprotection

.................... 
of focal cerebral ischemia employing intracerebrally applied endothelin-1 (6). These findings have been extended to models of transient global forebrain ischemia (7-10). FK506 is a fungal-derived macrolide exhibiting a potent immunosuppressant action, and has been recently introduced in clinical practice to prevent allograft rejection. The mechanism by which FK506 prevents ischemic brain damage is not understood, and may depend on de novo protein synthesis (11) rather than on the inhibition of calcineurin $(11,12)$. Since FK506 is already permitted for human use, and considering that it crosses the blood-brain barrier (13), it could be a promising candidate to enter clinical trials as a neuroprotective agent.

However, data obtained from animal models of global cerebral ischemia in vivo are still limited. Three studies employing FK506 have been performed using the gerbil model of global ischemia (7-9) and one study used the rat 2-vessel occlusion + hypotension model (10). The possible influence of drug-induced hypothermia in these studies is controversial. Only a single study has investigated whether the neuroprotective effects of FK506 are sustained for post-ischemic periods longer than the commonly used 4-7day survival time (7). This is an important aspect of neuroprotection since some treatments may merely delay, rather than prevent ischemia-induced cell loss (14).

There is a need for further investigation of the neuroprotective effects of FK506 in the rat, using the 4-vessel occlusion (4-VO) model (15). In an initial experiment, we attempted to reproduce a previous finding of robust neuroprotection in the gerbil after a single intravenous (iv) dose of FK506 (7). Given the unexpected negative results, subsequently we assessed the effects of FK506 applied in repeated doses, employing a combination of $i v$ and intraperitoneal ( $i p$ ) administration. The effects of FK506 were assessed at two different post-ischemic survival times, and evaluated in different rostro- caudal portions of the CA1 subfield. In a third experiment, we investigated improvement in FK506 neuroprotection by restricting the administration of repeated doses to the $i v$ route.

\section{Material and Methods}

\section{Animals}

Adult male Wistar rats weighing 270$300 \mathrm{~g}$ were housed in groups of three to four in plastic cages $(39 \times 33 \times 16 \mathrm{~cm})$ at controlled temperature $\left(22 \pm 1^{\circ} \mathrm{C}\right)$ on a 12 -h light/dark cycle (lights on at 7:00 h) with constant air exchange. Food and water were offered ad libitum. These housing conditions were maintained until the end of the experiments.

\section{Ischemia}

Transient global forebrain ischemia was induced by the 4-VO method (15) with modifications (16). Briefly, the animals were anesthetized with ether plus local application of $2 \%$ xylocaine, and were fixed in a stereotaxic-like frame; the vertebral arteries were then bilaterally electrocoagulated. Subsequently, the animals were placed in the supine position and the carotid arteries were carefully isolated from the adjacent tissues and loosely snared with a silk thread.

Five to six hours later the thread was gently tightened for a period of $15 \mathrm{~min}$. Loss of the righting reflex within 2 min of carotid occlusion, unresponsiveness to gentle touch, mydriasis, and tonic extension of the paws were considered to indicate effective ischemia. If loss of the righting reflex did not occur within the first 2 min of carotid occlusion, or if the righting reflex was recovered before the end of ischemia, the animal was excluded from the experiment. Throughout occlusion and during the first hour of reperfusion, the rats were maintained in a warming box at $30^{\circ} \mathrm{C}(17)$. Core temperature was 
monitored but not controlled during the ischemic period, and up to $3.5 \mathrm{~h}$ of reperfusion, using a rectal probe inserted to a depth of approximately $6 \mathrm{~cm}$.

Sham-operated animals were submitted to the same manipulations, except for vertebral and carotid occlusion. Experimental procedures followed the ethical principles established by the Brazilian College of Animal Experimentation (www.meusite.com.br/ COBEA/index.htm).

\section{Drug treatment}

In the first experiment, FK506 was administered $i v$ via the penile vein in single doses $(1.0,3.0$, or $6.0 \mathrm{mg} / \mathrm{kg}$, in a volume of $0.1 \mathrm{ml} / 100 \mathrm{~g}$ body weight) immediately after the beginning of reperfusion. In a second experiment, $1.0 \mathrm{mg}$ FK506/kg was given $i v$ at reperfusion (time $=0 \mathrm{~h}$ ) followed by ip injections applied 6, 24, 48 and 72 h postischemia. In the third study, repeated doses of FK506 $(1.0 \mathrm{mg} / \mathrm{kg})$ were administered only by the $i v$ route, but at the same intervals as in the second experiment. Control animals received vehicle alone $(0.1 \mathrm{ml} / 100 \mathrm{~g})$ as single or repeated injections where appropriate. Both FK506 (solution, $10 \mathrm{mg} / \mathrm{ml} \mathrm{am}$ poule) and vehicle (polyoxyethylene hydrogenated castor oil 60 and anhydrous ethanol) were kindly supplied by the Fujisawa Pharmaceutical Co., Osaka, Japan.

\section{Histological analysis}

Seven or thirty days after ischemia, the brain was removed for histological assessment of ischemic lesions in the hippocampus. The animals were deeply anesthetized with ether and perfused transcardiacally with $0.9 \%$ saline followed by Bouin's fixative $(20 \mathrm{ml} /$ $\min$ for 7-10 min). Following decapitation, the head was immersed in crushed ice $\left(1-2^{\circ} \mathrm{C}\right)$ for $1 \mathrm{~h}$. The brain was then carefully removed and kept in Bouin's fluid for 3 days.

In the first experiment (dose-response curve), ischemic damage was assessed at a level corresponding to approximately 4.52 $\mathrm{mm}$ posterior to bregma (18). Eight to twelve paraffin-embedded coronal sections $(5-\mu \mathrm{m}$ thickness) were taken from each brain and stained with celestine blue/acid fuchsin. Three sections from each stereotaxic level were chosen for bilateral counts of normalappearing neurons in the CA1 pyramidal stratum. Thus, six fields/level were counted for each rat, the number of cells in each level being expressed as the mean of the six fields. Fields were chosen by centering the $400 \mathrm{X}$ microscopic field laterally and close to the apex of the CA1 sectors in each respective level. The number of intact pyramidal cells with a distinct nucleus and nucleolus was counted along a transect of $450-\mu \mathrm{m}$ length (approximately $0.160 \mathrm{~mm}^{2}$ ). In subsequent experiments, the histological analysis was expanded to include two additional stereotaxic coronal planes, which corresponded approximately to 3.60 and $5.30 \mathrm{~mm}$ posterior to bregma. The identity of the treatment groups was not revealed during histological assessment.

\section{Statistical analysis}

The Kruskal-Wallis test was used to evaluate the effects of FK506 on ischemia-induced hippocampal CA1 neurodegeneration. In the second and third experiments, a separate test was used for data obtained at each stereotaxic level. When a significant main effect appeared, Dunn's multiple range test was performed to determine differences among the treatment groups. The effect of FK506 on rectal temperature was analyzed by MANOVA for repeated measures. In the case of a significant group effect, the Newman-Keuls multiple range test was used to distinguish the groups.

\section{Results}

Figure 1 shows the dose-effect relation- 
Figure 1. Dose-response effect of FK506 on hippocampal pyramidal CA1 cell loss caused by ischemia. FK506 $(1.0,3.0$ or $6.0 \mathrm{mg} / \mathrm{kg})$ was given iv immediately after the beginning of reperfusion. Hippocampal damage was assessed 7 days after ischemia and is expressed as a reduction in the number (mean \pm SEM) of intact-appearing pyramidal cells. Compared to the vehicletreated group (92.6\% cell loss), FK506 did not reduce CA1 damage, independently of the dose used ( $P>0.05$, Dunn's test). Each point represents one individual; the horizontal lines indicate the mean values of the respective groups. The numbers in parentheses indicate sample size. ship of FK506 on ischemia-induced CA1 cell death. Fifteen minutes of $4-\mathrm{VO}$ caused marked CA1 cell loss $(92.6 \%)(\mathrm{P}<0.001$, sham vs vehicle, Dunn's multiple comparison test). This neurodegenerative effect of ischemia was not mitigated by a single $i v$ dose of FK506, whatever the concentration used ( $\mathrm{P}>0.05$, vehicle $v s$ FK506). In contrast, repeated doses of FK506 were effective in reducing the neurodegenerative effect of ischemia (Figure 2A and B). Fifteen min-

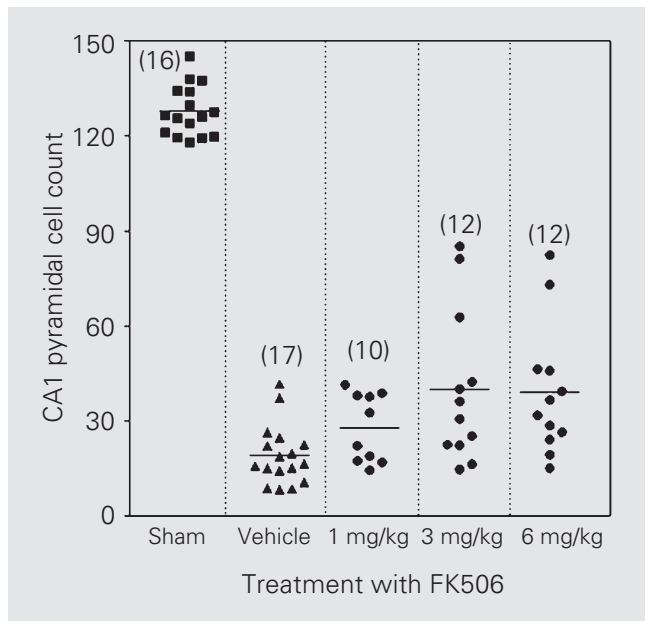

utes of 4-VO caused pronounced CA1 cell loss at all coronal levels analyzed (77.4$93.2 \%, \mathrm{P}<0.001$, sham $v s$ vehicle). When given repeatedly ( $1 i v$ injection $+4 i p$ injections), FK506 reduced the degree of cell death at all levels, independently of whether the animals were sacrificed 7 or 30 days post-ischemia $(\mathrm{P}<0.01-0.001$, vehicle $v s$ FK506, Dunn's multiple comparison test). In animals analyzed 30 days post-ischemia, neuroprotection was seen at the medial and temporal levels of the hippocampus (Figure $2 \mathrm{~B}: \mathrm{P}<0.05$, vehicle vs FK506), but not at the most rostral level $(\mathrm{P}>0.05)$. The degree of neuroprotection provided by FK506 varied as a function of the septotemporal level; this differential effect was clearly evident in the 7-day post-ischemic group (Figure $2 \mathrm{~A} ; \mathrm{F}_{1,40}$ $=12.36, \mathrm{P}=0.0011)$, but was reduced in the 30-day group (Figure 2B, $\mathrm{F}_{1,37}=3.64, \mathrm{P}=$ $0.064)$. Regression analysis for the vehicletreated groups also revealed that the severity of ischemia decreased from the septal to the temporal pole of the hippocampus in both the 7-day and 30-day post-ischemic groups (Figure 2A: $F_{1,19}=7.75, P=0.012$; Figure 2B: $\left.F_{1,25}=7.61 ; P<0.011\right)$. In the sham-

Figure 2. Effect of repeated iv plus ip doses of FK506 $(1.0 \mathrm{mg} / \mathrm{kg})$ on hippocampal pyramidal CA1 cell loss caused by ischemia. The first injection (iv) was given immediately after the beginning of reperfusion. Additional ip injections were given 6, 24, 48 and 72 h post-ischemia. Histological analysis was performed 7 (panel A) or 30 (panel B) days post-ischemia. Fifteen minutes of 4-vessel occlusion caused pronounced CA1 cell loss at all coronal levels analyzed $(P<0.001$, sham vs vehicle). FK506 reduced the degree of cell death in all stereotaxic planes studied, independently of whether the animals were sacrificed 7 or 30 days post-ischemia $(\mathrm{P}<0.01-0.001$, vehicle vs FK506, Dunn's test). The severity of ische-

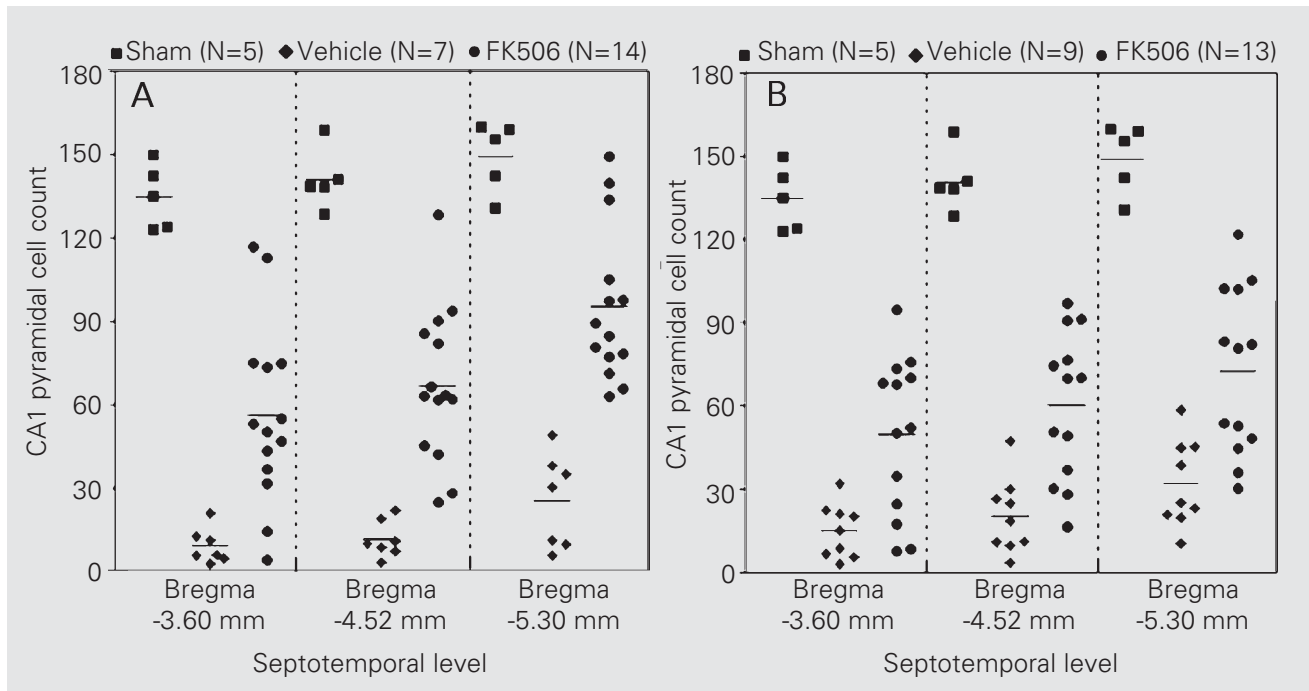

mia decreased (panel $A: P<0.05$; panel $B: P<0.011$ ), while FK506 neuroprotection increased (panel $A: P<0.005$; panel $B$ : $P=0.064$ ) along the septotemporal axis of the hippocampus. The sham-operated rats $(N=5)$ are the same in panels $A$ and $B$. 
operated group, the number of CA1 pyramidal neurons was unaltered among the various stereotaxic planes (linear regression: $\mathrm{P}>0.05$ ). Representative photomicrographs of the hippocampus of rats treated with vehicle or FK506 are illustrated in Figure 3.

Figure 4 shows the results of repeated $i v$ administration of FK506 $(1.0 \mathrm{mg} / \mathrm{kg})$, given $0,6,24,48$ and $72 \mathrm{~h}$ post-ischemia. As seen previously, $15 \mathrm{~min}$ of ischemia led to pronounced CA1 neurodegeneration at all septotemporal levels $(\mathrm{P}<0.01$, sham vs vehicle, Dunn's multiple comparison test), with the severity of ischemia decreasing from the septal to the temporal pole of the hippocampus $\left(\mathrm{F}_{1,25}=13.4, \mathrm{P}=0.0012\right)$. Repeated $i v$ injections of FK506 failed to reduce ischemia-induced $\mathrm{CA} 1$ cell death $(\mathrm{P}>0.05)$ at all septotemporal levels.

The effect of FK 506 on core temperature is illustrated in Figure 5. At all concentrations used, FK506 reduced rectal temperature compared to the vehicle group $(\mathrm{P}<0.05$ 0.001 , Newman-Keuls test). However, this effect did not alter the state of normothermia (approximately $37^{\circ} \mathrm{C}$ ), at least over the period during which temperature was monitored.
A
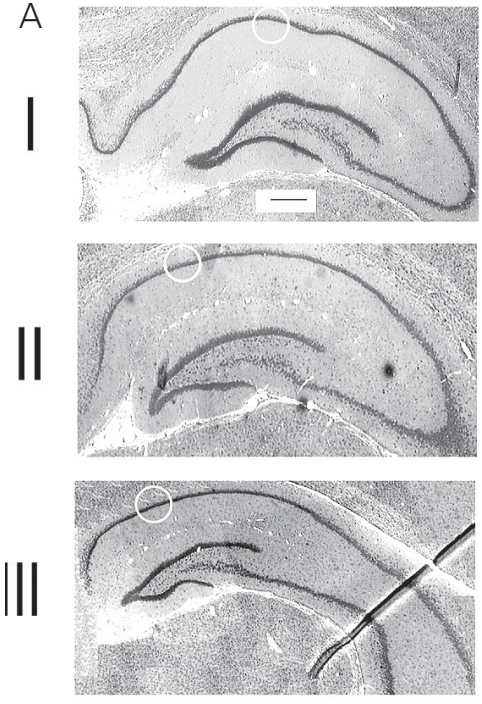

C

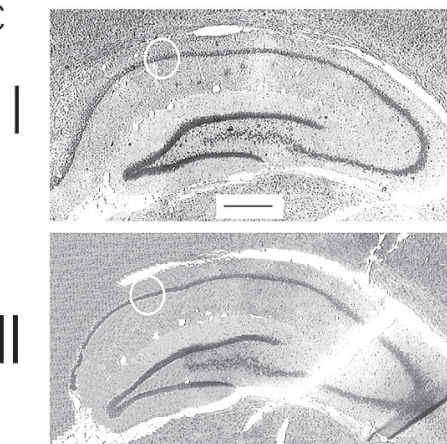

III

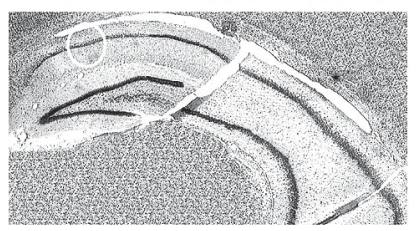

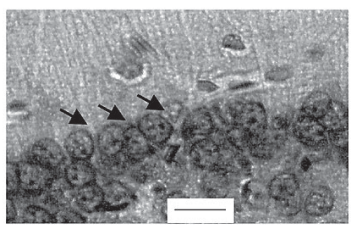
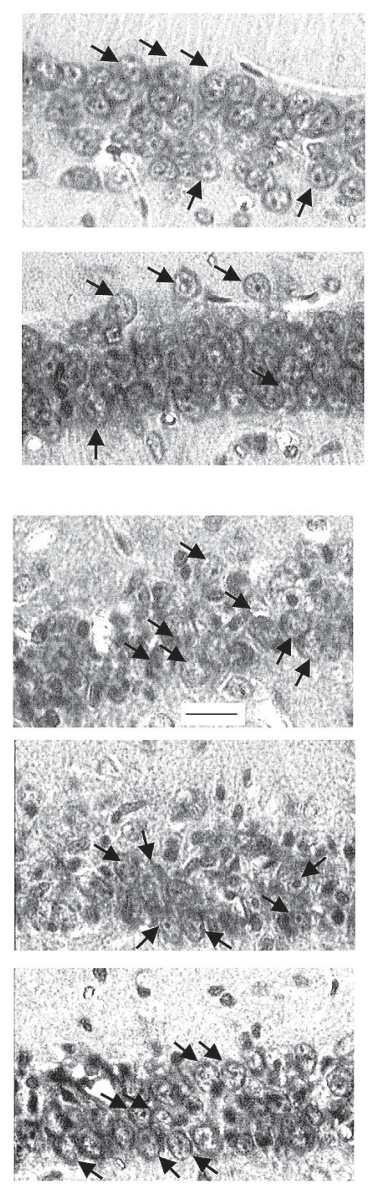

B
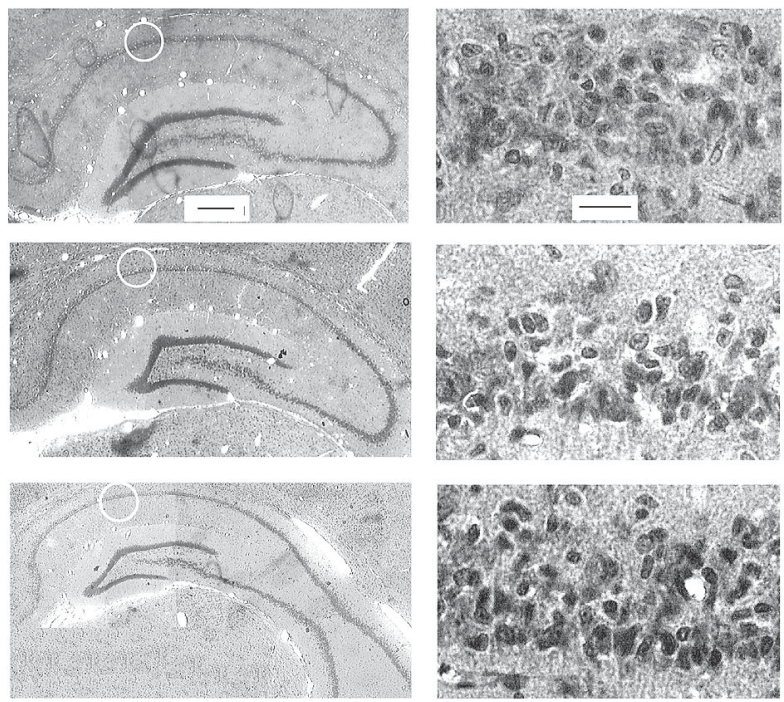

Figure 3. Photomicrographs of coronal sections of the hippocampus at stereotaxic levels corresponding to $-3.60 \mathrm{~mm}(\mathrm{I})$, $-4.52 \mathrm{~mm}$ (II) and $-5.30 \mathrm{~mm}$ (III) in rats subjected to sham operation (A), ischemia plus vehicle (B) or ischemia plus FK506 (C), at magnifications of 20X (left) and 400X (right). Hippocampal damage was assessed 7 days after ischemia. White circles (left panels) indicate the approximate location of the cell counts in the CA1 field. Intact-appearing pyramidal neurons are indicated by arrows. Bars $=100 \mu \mathrm{m}(20 \mathrm{X})$ and 20 $\mu \mathrm{m}(400 \mathrm{X})$. 


\section{Discussion}

The present study demonstrates that FK506 can attenuate hippocampal damage in models of transient global forebrain ischemia. Our data extend previous findings to the rat 4-VO model and suggest that such an effect may depend on a repeated dose protocol.

In the first experiment, a single intravenous injection of FK506 provided no neuro-

Figure 4. Effect of repeated doses of FK506 $(1.0 \mathrm{mg} / \mathrm{kg})$ iv on hippocampal pyramidal CA1 cell loss caused by ischemia. FK506 was given $0,6,24,48$ or $72 \mathrm{~h}$ post-ischemia. Histological analischemia. Fifteen minutes of 4vessel occlusion caused pronounced CA1 cell loss at all coronal levels analyzed $(P<0.01$, sham vs vehicle). Repeated iv injections of FK506 failed to prevent $\mathrm{CA} 1$ cell death at all coronal levels studied ( $P>0.05$, vehicle vs FK506, Dunn's test). ysis was performed 7 days post-
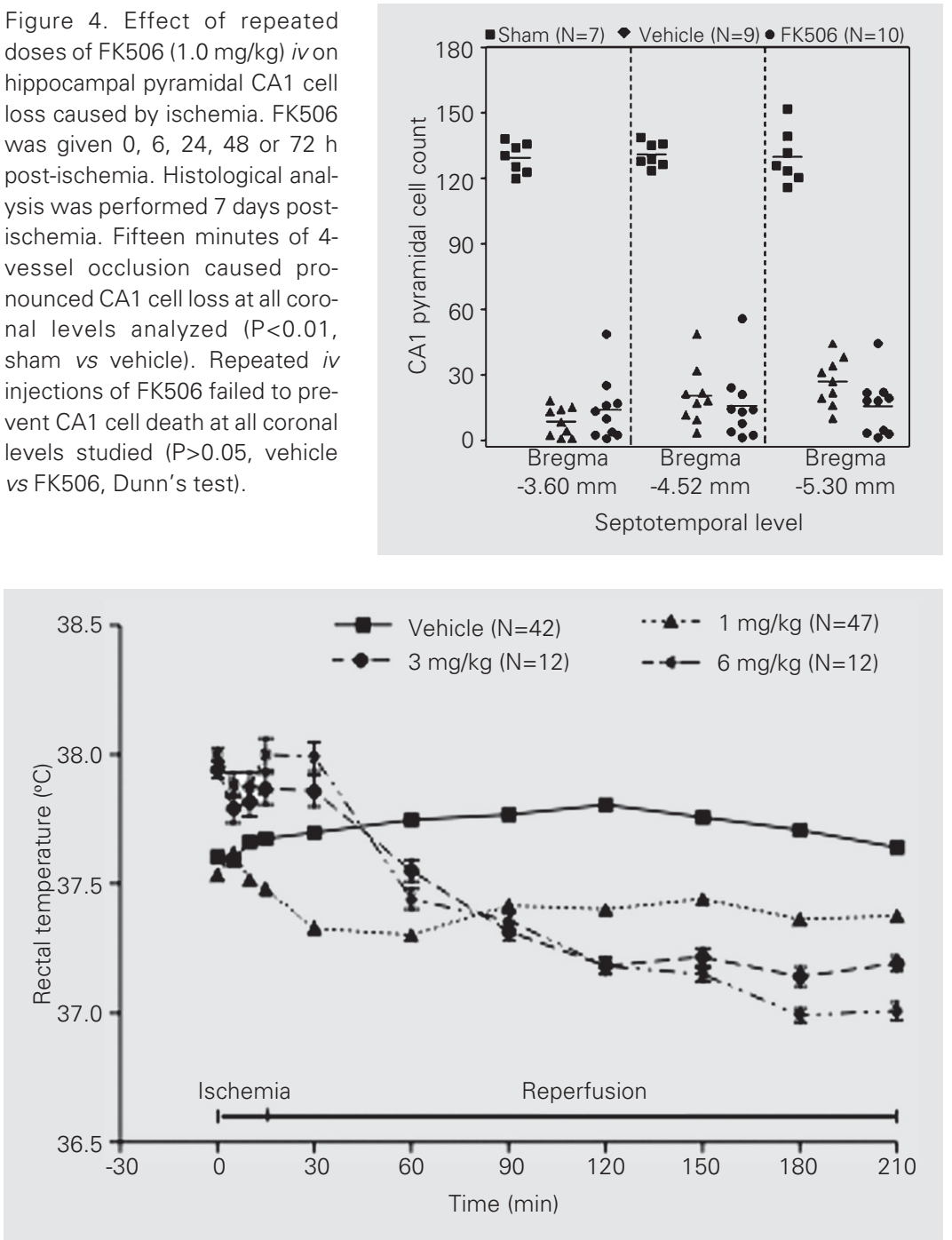

Figure 5. Change in rectal temperature (mean \pm SEM) of rats subjected to cerebral ischemia and treated with vehicle or FK506. The groups receiving vehicle or FK506 $(1 \mathrm{mg} / \mathrm{kg})$ in the three experiments were pooled, since rectal temperature was measured under the same treatment conditions. FK506 significantly reduced rectal temperature at all doses compared to the group receiving vehicle alone $(P<0.05-0.001$, Newman-Keuls test). However, this effect did not alter the degree of normothermia $\left(37-38^{\circ} \mathrm{C}\right)$. protection, whatever the concentration used $(1.0,3.0$, or $6.0 \mathrm{mg} / \mathrm{kg})$. This result does not confirm a previous finding that a single (3.0 or $10.0 \mathrm{mg} / \mathrm{kg}$ ) dose injected $i v$ immediately after ischemia resulted in robust neuroprotection of CA1 pyramidal cells (7). This discrepancy may be partially explained by the differences observed in the effect of FK506 on core temperature in each study. In the study by Ide et al. (7), FK506 caused a substantial and dose-dependent reduction in core temperature, i.e., $34^{\circ}$ and $32^{\circ} \mathrm{C}$, respectively. In the present experiment, FK506 did not induce hypothermia.

Significant and sustained neuroprotection was provided, however, when FK506 was given repeatedly, as a combination of $i v$ (1x) plus ip (4x) injections. Again, although rectal temperature was reduced by up to $1^{\circ} \mathrm{C}$ compared to vehicle-treated animals, this decrease was far from that at which hypothermia is considered to be neuroprotective, i.e., less than $33^{\circ} \mathrm{C}$. Thus, the neuroprotective effect does not appear to be due to the influence of FK506 on core temperature. This finding agrees with other studies. In the gerbil, a single injection of FK506 (1.0 mg/ $\mathrm{kg}$ ) ip did not prevent ischemia-induced CA1 cell death. In contrast, the same dose given daily for four days provided neuroprotection, without hypothermia during the $24 \mathrm{~h}$ following FK506 administration $(8,9)$. In rats, FK506 prevented ischemia-induced brain damage without reducing core temperature when given daily for 4 (10) or 14 days (19). In the present study, rectal temperature was recorded for up to $3.5 \mathrm{~h}$ post-ischemia (Figure 5); we cannot rule out the possibility that hypothermia may have occurred subsequent to FK506 given $6,24,48$ or $72 \mathrm{~h}$ postischemia.

In the present study, the neuroprotective efficacy of FK506 increased from the septal to the temporal pole of the hippocampus. In contrast, the severity of ischemia declined slightly but significantly along the same axis. This may influence the degree of neuropro- 
tection by FK506 in an inverse fashion, i.e., FK506 prevented CA 1 cell death most effectively in the region of the hippocampus least sensitive to ischemia. Similar results have been observed following hypothermia in the gerbil model (20). Differential sensitivity of CA1 pyramidal cells to ischemia, decreasing from the septal to the temporal pole of the hippocampus, has been demonstrated by others $(21,22)$. Since sensitivity to ischemia may vary within the same brain region, the neuroprotective efficacy of drugs will not necessarily be the same in different parts of the brain, or even within the same brain structure. In this respect, our data are interesting given that the hippocampus is functionally differentiated into several parts, and that behavioral impairments following hippocampal lesion also vary following a septotemporal lesion gradient (23). Our observations thus emphasize the importance of a multilevel histological analysis associated with behavioral measurements when assessing the neuroprotective efficacy of drugs in future studies.

A major question in neuroprotection refers to the maintenance of the neuroprotective effect over time. In the present study, the neuroprotective effect of FK506 was sustained at least up to 30 days after the beginning of treatment; however, neuroprotection was less than, but not statistically different from, that measured 7 days after ischemia. Sustained neuroprotection for approximately 30 days of recovery has also been obtained with benzodiazepine-related compounds (24) and felbamate (25) in the gerbil, and after mild hypothermia in the rat (26). In contrast, the neuroprotective effect of the GABA reuptake inhibitor tiagabine is evident at 4 days, but not after 21 days of recovery (14). These findings emphasize the importance of considering different post-ischemic survival times when the neuroprotective efficacy of drugs is evaluated under conditions of cerebral ischemia.

Despite the neuroprotective effect of
FK506 following repeated and combined $i v$ plus ip injections (Figure 2), it must be emphasized that some animals showed no benefit from FK506. Such a "bimodal" distribution of ischemic CA1 damage has also been reported after treatment with different compounds, such as the AMPA receptor antagonist, NBQX $(27,28)$, the benzodiazepine agonist, diazepam $(24,29,30)$, and the partial benzodiazepine agonist, imidazenil (24). Such variability in response is thought to result from a combination of at least two factors, i.e., normal variation of damage in the model, and differences in the real concentration of the drug which reaches the brain in each individual (27). We considered this last factor in the present study, and the third experiment (Figure 4) was designed to test whether FK506 efficacy could be increased when all five doses were administered by the $i v$ route alone. The rationale was that by increasing FK506 bioavailability, greater neuroprotective efficacy might be provided. Unexpectedly, however, no neuroprotection was obtained using this schedule. At present, we have no explanation for this finding.

In conclusion, the present study further demonstrates that FK506 reduces the extent of neuronal death in the hippocampus after transient global cerebral ischemia in rats, and extends to the rat 4-VO model previous observations that FK506 protects against ischemia in a sustained manner. This effect may depend on a schedule of repeated doses via different delivery routes. However, more detailed studies are needed to assess the most appropriate treatment protocol that provides the greatest efficacy of FK506. Additional studies are also required to investigate the therapeutic window of the neuroprotective effect of FK506. At present, we are investigating whether the neuroprotective effect of FK506 observed at the histological level is accompanied by attenuation of ischemia-induced, spatial learning and memory dysfunction. 


\section{Acknowledgments}

The authors gratefully acknowledge the technical assistance of Ricardo A. Paganelli (computer-assisted images) and Marcos A. Trombelli (animal care).

\section{References}

1. Zola-Morgan S, Squire LR \& Amaral DG (1986). Human amnesia and the medial temporal region: enduring memory impairment following a bilateral lesion limited to field CA1 of the hippocampus. Journal of Neuroscience, 6: 2950-2967.

2. Petito CK, Feldmann E, Pulsinelli WA \& Plum F (1987). Delayed hippocampal damage in humans following cardiorespiratory arrest. Neurology, 37: 1281-1286.

3. Pulsinelli WA, Brierley JB \& Plum F (1982). Temporal profile of neuronal damage in a model of transient forebrain ischemia. Annals of Neurology, 11: 491-498.

4. Kirino T, Tamura A \& Sano K (1984). Delayed neuronal death in the rat hippocampus following transient forebrain ischemia. Acta Neuropathologica, 64: 139-147.

5. Dawson TM, Steiner JP, Dawson VL, Dinerman JL, Uhl GR \& Snyder SH (1993). Immunosuppressant FK506 enhances phosphorylation of nitric oxide synthase and protects against glutamate neurotoxicity. Proceedings of the National Academy of Sciences, USA, 90: 9808-9812.

6. Sharkey J \& Butcher SP (1994). Immunophilins mediate the neuroprotective effects of FK506 in focal cerebral ischaemia. Nature, 371: 336-339.

7. Ide T, Morikawa E \& Kirino T (1996). An immunosuppressant, FK506, protects hippocampal neurons from forebrain ischemia in the Mongolian gerbil. Neuroscience Letters, 204: 157-160.

8. Tokime T, Nozaki K \& Kikuchi H (1996). Neuroprotective effect of FK506, an immunosuppressant, on transient global ischemia in gerbil. Neuroscience Letters, 206: 81-84.

9. Yagita Y, Kitagawa K, Matsushita K, Taguchi A, Mabuchi T, Ohtsuki T, Yanagihara T \& Matsumoto M (1996). Effect of immunosuppressant FK506 on ischemia-induced degeneration of hippocampal neurons in gerbils. Life Sciences, 59: 1643-1650.

10. Drake M, Friberg H, Boris-Moller F, Sakata K \& Wieloch T (1996). The immunosuppressant FK506 ameliorates ischaemic damage in the rat brain. Acta Physiologica Scandinavica, 158: 155-159.

11. Klettner A, Baumgrass R, Zhang Y, Fischer G, Burger E, Herdegen T \& Mielke $K$ (2001). The neuroprotective actions of FK506 binding protein ligands: neuronal survival is triggered by de novo RNA synthesis, but is independent of inhibition of JNK and calcineurin. Molecular Brain Research, 97: 21-31.

12. Morioka M, Fukunaga K, Kai Y, Todaka T, Yano S, Hamada J-I, Miyamoto E \& Ushio Y (2001). Intravenously injected FK506 failed to inhibit hippocampal calcineurin. Biochemical and Biophysical Research Communications, 286: 802-806.

13. Yoshimoto T \& Siesjo BK (1999). Posttreatment with the immunosuppressant cyclosporin A in transient focal ischemia. Brain Research, 839: 283-291.

14. Inglefield JR, Perry JM \& Schwartz RD (1995). Postischemic inhibition of GABA reuptake by tiagabine slows neuronal death in the gerbil hippocampus. Hippocampus, 5: 460-468.

15. Pulsinelli WA \& Brierley JB (1979). A new model of bilateral hemispheric ischemia in the unanesthetized rat. Stroke, 10: 267-272.

16. Milani H, Lepri ER, Giordani F\& Favero-Filho LA (1999). Magnesium chloride alone or in combination with diazepam fails to prevent hippocampal damage following transient forebrain ischemia. Brazilian Journal of Medical and Biological Research, 32: 1285-1293.

17. Seif el Nasr M, Nuglish J \& Krieglstein J (1992). Prevention of ischemia-induced cerebral hypothermia by controlling the environmental temperature. Journal of Pharmacological Methods, 27: 2326.

18. Paxinos G \& Watson C (1986). The Rat Brain in Stereotaxic Coordinates. 2nd edn. Academic Press, Orlando, FL, USA.

19. Wakita H, Tomimoto H, Akiguchi I \& Kimura J (1998). Dose-dependent, protective effect of FK506 against white matter changes in the rat brain after chronic cerebral ischemia. Brain Research, 792: 105113.

20. Corbett D, Nurse S \& Colbourne F (1997). Hypothermic neuroprotection. A global ischemia study using 18- to 20-month-old gerbils. Stroke, 28: 2238-2243.

21. Crain BJ, Westerkam WD, Harrison AH \& Nadler JV (1988). Selective neuronal death after transient forebrain ischemia in the Mongolian gerbil: A silver impregnation study. Neuroscience, 27: 387-402.

22. Auer RN, Jensen ML \& Whishaw IQ (1989). Neurobehavioral deficit due to ischemic brain damage limited to half of the CA1 sector of the hippocampus. Journal of Neuroscience, 9: 1641-1647.

23. Moser M-B \& Moser El (1998). Functional differentiation in the hippocampus. Hippocampus, 8: 608-619.

24. Schwartz-Bloom RD, McDonough KJ, Chase PJ, Chadwick LE, Inglefield JR \& Levin ED (1998). Long-term neuroprotection by benzodiazepine full versus partial agonists after transient cerebral ischemia in the gerbil. Journal of Cerebral Blood Flow and Metabolism, 18: 548-558.

25. Shuaib A, Waqaar $T$, ljaz MS, Kanthan R, Wishart $T$ \& Howlett $W$ (1996). Neuroprotection with felbamate: a 7- and 28-day study in transient forebrain ischemia in gerbils. Brain Research, 727: 65-70.

26. Colbourne F, Li H \& Buchan AM (1999). Indefatigable CA1 sector neuroprotection with mild hypothermia induced $6 \mathrm{~h}$ after severe forebrain ischemia in rats. Journal of Cerebral Blood Flow and Metabolism, 19: 742-749.

27. Buchan AM, Li H, Cho S \& Pulsinelli WA (1991). Blockade of the AMPA receptor prevents CA1 hippocampal injury following severe but transient forebrain ischemia in adult rats. Neuroscience Letters, 132: 255-258.

28. Li H \& Buchan AM (1993). Treatment with an AMPA antagonist $12 \mathrm{~h}$ following severe normothermic forebrain ischemia prevents CA1 neuronal injury. Journal of Cerebral Blood Flow and Metabolism, 13: 933-939.

29. Schwartz RD, Yu X, Katzman MR, Hyden-Hixson DM \& Perry JM (1995). Diazepam, given postischemia, protects selectively vulnerable neurons in the hippocampus and striatum. Journal of Neuroscience, 15: 529-539.

30. Dowden J, Reid C, Dooley P \& Corbett D (1999). Diazepam-induced neuroprotection: dissociating the effects of hypothermia following global ischemia. Brain Research, 829: 1-6. 